Nota Científica

\title{
Primer reporte de infección causada por el protozoario Ophryocystis elektroscirrha McLaughlin y Myers (Neogregarinida: Ophryocystidae) en la mariposa monarca Danaus plexippus megalippe f. portoricensis A. Clark (Lepidoptera: Nymphalidae) en Puerto Rico
}

First report of infection caused by the protozoan Ophryocystis elektroscirrha McLaughlin \& Myers (Neogregarinida: Ophryocystidae) in the monarch butterfly Danaus plexippus megalippef. portoricensis A. Clark (Lepidoptera: Nymphalidae) in Puerto Rico

Ramón A. Rosario-Rodríguez ${ }^{1,2}$ y Carlos A. Acevedo-Suárez ${ }^{1}$ (D)

${ }^{1}$ Departamento de Biología, Universidad de Puerto Rico-Mayagüez, Call Box 9000 Mayagüez, PR 00681.

险carlos.acevedo5@upr.edu

${ }^{2}$ Division of Science, Technology, Engineering, and Math (STEM), Reading Area Community College, $10 \mathrm{~S}$ 2nd St, Reading, PA 19603. E-mail: rrosariorodriguez@racc.edu

\section{ZooBank: urn:lsid:zoobank.org:pub: D0B07CBF-24F1-49B5-9417-E2BE3139F816 \\ https: / / doi.org/10.35249/ rche.46.3.20.18}

Resumen. Se registran por primera vez en Puerto Rico infecciones causadas por el protozoario Ophryocystis elektroscirrha McLaughlin y Myers, 1970 en especímenes de la mariposa monarca Danaus plexippus megalippe f. portoricensis A. Clark, 1941. Se encontraron esporas del parásito en escamas de varios individuos provenientes del mariposario del zoológico Dr. Juan A. Rivero en Mayagüez, al oeste de la isla. Se concluye que la presencia del protozoario no es rara y que O. elektroscirrha probablemente no había sido detectado anteriormente por la falta de estudios de la mariposa monarca en Puerto Rico. Se proveen figuras que permiten la identificación del parásito.

Palabras clave: Enfermedad, esporas, no migratoria, parásito.

Abstract. Infections with the protozoan Ophryocystis elektroscirrha McLaughlin \& Myers, 1970 are reported for the first time in specimens of the monarch butterfly, Danaus plexippus megalippe $f$. portoricensis A. Clark, 1941 in Puerto Rico. Spores from the parasite were found on the scales of several individuals from the butterfly garden at Dr. Juan A. Rivero Zoo in Mayagüez, on the western side of the island. It is concluded that the presence of the protozoan is not rare and that O. elektroscirrha has probably not been detected previously due to the lack of studies of the monarch butterfly in Puerto Rico. Figures are provided to allow for the identification of the parasite.

Key words: Disease, non-migratory, parasite, spores.

La mariposa monarca, Danaus plexippus Linnaeus, 1758 (Lepidoptera: Nymphalidae: Danainae), es conocida por su extensa migración a través de Estados Unidos y México, aunque también se puede encontrar en otros lugares del continente americano (Brower 1996; Brower y Malcolm 1991; Pierce et al. 2015). La supervivencia de la especie se considera amenazada por la pérdida de hábitat, uso de plaguicidas, depredación y el cambio climático (Flockhart

Recibido 14 Julio 2020 / Aceptado 2 Septiembre 2020 / Publicado online 25 Septiembre 2020 Editor Responsable: José Mondaca E. 
et al. 2015). Las subespecies de mariposa monarca se pueden dividir en migratorias y no migratorias. En Puerto Rico se puede encontrar a Danaus plexippus megalippe f. portoricensis A. Clark, 1941, la forma local de una subespecie no migratoria que presenta una coloración endémica de la isla (Pérez-Asso et al. 2009). En ocasiones se menciona en la literatura como Danaus plexippus portoricensis (e.g., Echevarría Ramos y Hulshof 2019), pero seguimos la clasificación propuesta por Smith et al. (2005).

El protozoario Ophryocystis elektroscirrha McLaughlin y Myers, 1970 (Neogregarinida: Ophryocystidae) (referido en adelante como OE) es un parásito obligado que infecta a la mariposa monarca (McLaughlin y Myers 1970; Leong et al. 1997; Altizer y Oberhauser 1999; Altizer 2001) tanto en subespecies migratorias como no migratorias. Es transmitido por las mariposas hembras a su progenie mediante esporas transferidas del cuerpo a los huevos durante la oviposición. Las esporas son ingeridas por las larvas y cuando emergen los adultos, se encuentran cubiertos de esporas, principalmente en el abdomen, permitiendo así que se repita el ciclo de vida del parásito. La infección reduce la expectativa de vida de las mariposas afectadas y puede resultar en la inhabilidad de eclosionar de los nuevos individuos.

Se obtuvieron muestras del abdomen de varios especímenes adultos de la subespecie de monarca de Puerto Rico del zoológico Dr. Juan A. Rivero en Mayagüez. Las poblaciones del mariposario del zoológico se componen de individuos criados en cautiverio y de otros capturados en el Refugio Nacional de Vida Silvestre de Cabo Rojo, Puerto Rico (parte del Servicio de Pesca y Vida Silvestre de los Estados Unidos). Las muestras se examinaron utilizando microscopía electrónica de barrido. Estos individuos presentaban algunos síntomas de infección con OE incluyendo que no sobrevivieron por más de una hora después de eclosionar y que no pudieron extender sus alas. Se observaron esporas elípticas en las escamas de los individuos examinados (Fig. 1). Su morfología (incluyendo forma, color y tamaño) es consistente con lo que ha sido previamente reportado para OE (McLaughlin y Myers 1970; Vickerman et al. 1999; Sternberg et al. 2012). Entendemos que esta es la primera observación de infección con $\mathrm{OE}$ en la mariposa monarca de Puerto Rico. Este hallazgo demuestra que las mariposas monarcas migratorias y no migratorias enfrentan retos similares para su supervivencia en Puerto Rico.

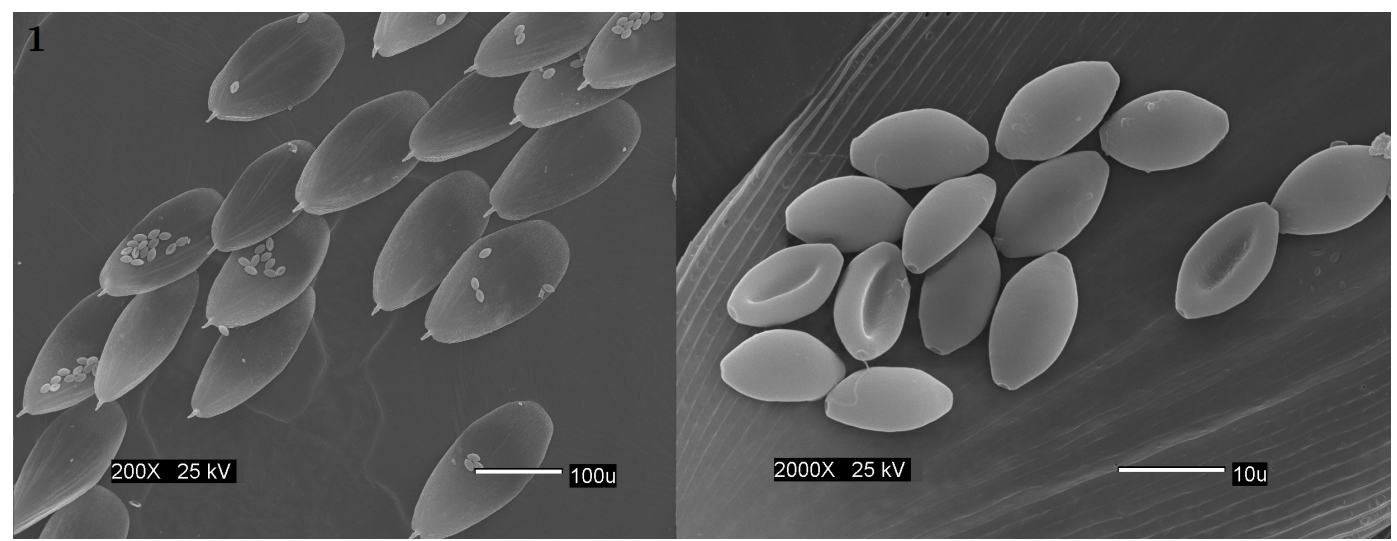

Figura 1. Imágenes de microscopía electrónica de barrido correspondientes a esporas de OE en escamas abdominales de especímenes adultos de Danaus plexippus megalippe f. portoricensis.

\section{Agradecimientos}

Agradecemos a Joalisse Mendoza Ramos, del zoológico Dr. Juan A. Rivero en Mayagüez por proveernos especímenes de la mariposa monarca, y a José R. Almodóvar Rivera, del Centro de Microscopía del Departmento de Biología de la Universidad de Puerto RicoMayagüez por su ayuda técnica. 


\section{Literatura Citada}

Altizer, S. (2001) Migratory behaviour and host - parasite co-evolution in natural populations of monarch butterflies infected with a protozoan parasite. Evolutionary Ecology Research, 3: 611-632.

Altizer, S.y Oberhauser, K. (1999) Effects of the protozoan parasite Ophryocystis elektroscirrha on the fitness of monarch butterflies (Danaus plexippus). Journal of Invertebrate Pathology, 88: 76-88.

Brower, L. (1996) Monarch butterfly orientation: Missing pieces of a magnificent puzzle. Journal of Experimental Biology, 199: 93-103.

Brower, L.P. y Malcolm, S.B. (1991) Animal migrations: Endangered phenomena. American Zoologist, 31: 261-276.

Echevarría Ramos, M. y Hulshof, C.M. (2019) Using digitized museum collections to understand the effects of habitat on wing coloration in the Puerto Rican monarch. Biotropica, 51: 477-483.

Flockhart, D.T.T., Pichancourt, J-B., Norris, D.R. y Martin, T.G. (2015) Unravelling the annual cycle in a migratory animal: breeding-season habitat loss drives population declines of monarch butterflies. Journal of Animal Ecology, 84: 155-165.

Leong, K.L.H., Yoshimura, M.A., Kaya, H.K. y Williams, H. (1997) Instar susceptibility of the monarch butterfly (Danaus plexippus) to the neogregarine parasite, Ophryocystis elektroscirrha. Journal of Invertebrate Pathology, 69: 79-83.

McLaughlin, R.E. y Myers, J.H. (1970) Ophryocystis elektroscirrha sp. n., a neogregarine pathogen of the monarch butterfly Danaus plexippus (L.) and the Florida queen butterfly D. gilippus berenice Cramer. Journal of Protozoology, 17: 300-305.

Pérez-Asso, A.R., Genaro, J.A. y Garrido, O.H. (2009) Butterflies of Puerto Rico - Las Mariposas de Puerto Rico. Editorial Cocuyo. San Juan, Puerto Rico. 140 pp.

Pierce, A.A., Altizer, S., Chamberlain, N.L., Kronforst, M.R. y de Roode, J.C. (2015) Unraveling the mysteries of monarch migration and global dispersal through molecular genetic techniques. Pp. 257-267. In: Oberhauser, K., Nail, K.R. y Altizer, S. (Eds.) Monarchs in a changing world: Biology and conservation of an iconic butterfly. Cornell University Press, New York.

Smith, D.A., Lushai, G. y Allen, J.A. (2005) A classification of Danaus butterflies (Lepidoptera: Nymphalidae) based upon data from morphology and DNA. Zoological Journal of the Linnean Society, 144: 191-212.

Sternberg, E.D., Lefevre, T., Li, J., de Castillejo, C.L.F., Li, H., Hunter, M.D. y de Roode, J.C. (2012) Food plant derived disease tolerance and resistance in a natural butterflyplant-parasite interaction. Evolution, 66: 3367-3376.

Vickerman, D., Michels, A. y Burrowes, P. (1999) Levels of infection of migrating monarch butterflies, Danaus plexippus (Lepidoptera: Nymphalidae) by the parasite Ophryocystis elektroscirrha (Neogregarinida: Ophryocystidae), and evidence of a new mode of spore transmission between adults. Journal of the Kansas Entomological Society, 72: 124-128. 